\title{
Development And Evaluation Of An Interactive Multimedia Clinical Skills Teaching Program Designed For The Pediatric Clerkship.
}

\author{
Anthony J. Frisby, $\mathrm{PhD}$, \\ Academic Information Services and Research \\ Thomas Jefferson University, Philadelphia, PA, USA \\ J. Lindsey Lane, MD, \\ Department of Pediatrics \\ Jefferson Medical College, Philadelphia, PA, USA \\ Anna Marie Carr, MD, \\ Department of Pediatrics \\ Albert Einstein Medical Center, Philadelphia, PA, USA \\ Ellen Ross, MD \\ Department of Pediatrics \\ Jefferson Medical College, Philadelphia, PA, USA \\ Ruth P. Gottlieb, MD \\ Department of Pediatrics \\ Jefferson Medical College, Philadelphia, PA, USA
}




\section{Background}

The traditional medical education program does very little to prepare medical students for their first encounter with pediatric patients. This can be particularly stressful for students whose first clerkship rotation is pediatrics. As the pediatric clerkship faculty at Jefferson Medical College had been observing students using structured clinical observations (SCOs) ${ }^{(1)}$ and videotaping ${ }^{(2)}$ those students it became clear that the students lacked basic history taking and physical examination skills. A more systematic approach was needed, to ensure a standard level of initial training and preparation in these two clinical skill areas for all students. A search for any existing materials-print, video or multimedia-failed to produce the type of coverage and training the clerkship directors sought.

The Academic \& Instructional Support \& Resources (AISR) staff provides both instructional support and software program development for faculty at Jefferson. AISR has

produced a variety of popular training materials for medical student and resident education. ${ }^{(3)}$ Clerkship faculty met with AISR staff to inquire about producing a program to address the unique needs of pediatric clerkship students in the areas of physical examination and history taking.

A development team was assembled which included: the clerkship directors, an instructional designer, programmer, videographer, graphic artist and a project manager. Because the pediatric clerkship faculty had been directly observing and videotaping students for the past few years, many hours of tapes of student performance, as well as data from a direct observation program, were available to review. After reviewing and identifying the common errors or omissions, the clerkship directors outlined the key components of the history and physical examination they wanted students to learn. Working with the design team, the content faculty 
developed the script and produced storyboards for the video recording along with necessary graphics and animation sequences.

Once the program, The Infant Patient Encounter, was developed it was field-tested during several clerkships rotations. Adjustments were made to areas the students found confusing or lacked the details that the students felt were important. There are many descriptive and demonstration articles on computer assisted instruction in the literature; but fewer

performance evaluation studies. ${ }^{(4)}$ Therefore, once the new version was completed we wanted to determine if the program would have the desired effect of improving student performance, not only student satisfaction.

\section{Purpose}

To measure the effect on performance of a multimedia program designed to teach medical students an infant physical exam.

\section{Methods}

The multimedia program has two distinct parts, history taking and physical examination. A convenience sample of $3^{\text {rd }}$ year students enrolled at Jefferson Medical College rotating through the six-week pediatric clerkship at Thomas Jefferson University Hospital, Albert Einstein Medical Center in Philadelphia, or the duPont Hospital for Children in Delaware, participated in the study. The clerkship directors at each of these institutions meet regularly to review and maintain a common curriculum. Students at each of the clerkship sites had the same teaching sessions and similar clinical experiences, and none of the sites offered any special program 
aimed at teaching physical examination skills. The study took place in the 2002-3 academic year.

On the first day of the clerkship, Jefferson and Einstein students were randomly assigned to one of two groups: physical exam (PX) or history taking (HX). Students' baseline physical examination skills were assessed and because of time and logistical limitations it was decided to observe part of the physical examination rather than a complete examination. The abdominal examination was selected to represent the full physical exam because it is a basic core competency for pediatrics. The abdominal exam is performed similarly on patients in other age groups (transferable), is relatively easy to observe without disrupting the student, and well tolerated by infants. A checklist was developed by the clerkship directors from each of the study sites based on the content of the program. The checklist was field tested and the three faculty raters found it easy to use and score. Students examined a standardized patient (a baby with the mother present) while faculty in the room observed the student's abdominal examination and used the checklist to rate student performance. (Appendix A). Points were awarded for correctly completed items. Coding of attempted but incorrectly executed items was done in order to identify the common mistakes students made - but no score was given for improperly performed components. Funding to pay the families for their participation came from the Jefferson Medical College Dean's office.

Two versions of the Infant Patient Encounter were created: one that only presented the physical-examination section and a second that presented only the history-taking section. Following the first infant exam, students had 1.5 hours to complete their assigned version of the program. After completion of the intervention, each student performed a second infant abdominal examination and the same faculty member using the checklist rated the performance. 
The faculty members who rated the students were blinded as to whether a student was in the intervention $(P X, n=20)$ group or the non-intervention $(H X, n=20)$ group.

Students were not allowed access to the program again during their clerkship. After the six-week clerkship, the original students in the study (HX and PX groups) and a control group (CX), from duPont Hospital for Children, were required to perform a final infant exam and were scored using the same checklist. The CX group (n=31) was unaware of the existence of the program and did not know in advance that they would be required to demonstrate an abdominal examination. Even though the faculty members were blinded to each group, they might have recognized the control $(\mathrm{CX})$ group because they had not participated in the first part of the study. For most sessions two faculty members rated each student and agreed on a final score. For some sessions only one rater was available. Students who were unavailable to participate in all test sessions for their group were excluded from the analysis.

The initial plan for analysis was to perform a 2 groups x 3 occasions ANOVA. However, at post clerkship, only $n=20$ of 28 in PX group and $n=20$ of 30 in HX group responded. To determine the effect of loss to follow up, 2 × 2 ANOVAs were performed on the full dataset $(\mathrm{n}=$ 28 and 30) and on dataset with missing data $n=20$ and 20) for baseline and post intervention data. The $2 \times 2$ ANOVAs showed similar results, so the $2 \times 3$ ANOVA was performed on the latter two groups ( $\mathrm{n}=20$ and 20$)$ across all three testing occasions - baseline, post intervention, and post clerkship - and those results are presented here. Standardized mean differences, d values, were computed to evaluate the magnitude of the effects of the intervention over occasions. A one-way ANOVA with three levels and Tukey's follow up tests were used to compare the PX and HX groups with the Control group at post clerkship. 
This study was determined to be exempt from IRB review by Thomas Jefferson University's Division of Human Subjects Protection.

\section{Results}

The baseline means for the PX and HX groups were not significantly different $(\mathrm{p}=$ .9373). (See Table 1). The mean of the PX group increased significantly from baseline to post intervention $(2.50$ to $6.80, \mathrm{~d}=2.15, \mathrm{p}=.0001)$. For the HX group, the difference from baseline to post intervention was not significant $(\mathrm{p}=.1488)$. The PX group mean was significantly higher than HX group mean at post intervention (6.80 versus $3.10, \mathrm{~d}=2.01, \mathrm{p}=.0001)$.

From post intervention to post clerkship, the mean of the PX group decreased significantly (6.80 to 5.50, $\mathrm{d}=-.54, \mathrm{p}=.0124)$, and the mean of the HX group increased significantly (3.01 to $4.40, \mathrm{~d}=.64, \mathrm{p}=.0226)$. The post clerkship PX and HX means were not significantly different at post clerkship (5.50 versus $4.40, \mathrm{~d}=43, \mathrm{p}=.1829)$. The mean of the Control group - obtained post clerkship only - was significantly lower than PX and HX means, but was similar to their baseline means (2.71 versus 2.50 and 2.75 ). 


\section{Table 1}

\begin{tabular}{|c|c|c|c|c|c|c|c|c|c|}
\hline \multirow[b]{2}{*}{ Group } & \multirow[b]{2}{*}{$\mathbf{n}$} & \multicolumn{2}{|c|}{ Baseline } & \multicolumn{2}{|c|}{$\begin{array}{l}\text { Post } \\
\text { Intervention }\end{array}$} & \multicolumn{2}{|c|}{$\begin{array}{l}\text { Post } \\
\text { Clerkship }\end{array}$} & \multicolumn{2}{|l|}{ d } \\
\hline & & Mean & (SD) & Mean & (SD) & Mean & (SD) & $\begin{array}{l}\text { Baseline/ } \\
\text { Post Intervention }\end{array}$ & $\begin{array}{l}\text { Post } \\
\text { Intervention/ } \\
\text { Post Clerkship }\end{array}$ \\
\hline PX & 20 & 2.50 & $(1.76)$ & 6.80 & $(2.21)$ & 5.50 & $(2.59)$ & $2.15^{*}$ & $-.54 *$ \\
\hline HX & 20 & 2.75 & (1.41) & 3.10 & (1.37) & 4.40 & $(2.54)$ & .25 & $.64 *$ \\
\hline Control & 31 & & & & & 2.71 & $(1.51)$ & & \\
\hline $\begin{array}{l}\text { Effect Sizes: } \\
* \mathrm{p}<.05\end{array}$ & PX/HX & -.16 & & $2.01 *$ & & .43 & & & \\
\hline
\end{tabular}

\section{Discussion}

Our study shows that The Infant Patient Encounter program was effective, compared to routine clerkship teaching, in improving physical examination (PE) skills. Of particular interest is that by the end of the clerkship our history-taking control group improved their skills almost as much as the PX intervention group. We speculate that this was because they responded to being evaluated at the beginning of the clerkship and sought out the information and skills about the physical exam from the PX group of students or from the faculty. The control group that was geographically separated was not evaluated at the beginning of the clerkship and did not reflect this phenomenon.

When asked on the end of clerkship evaluation form, $30 \%$ of the PX group reported that they received no instruction on physical examination during the clerkship compared to $23 \%$ of the HX group and $40 \%$ of the CX group. Although we do not know the extent or quality of that instruction, it might have improved the HX group performance at the end of the clerkship.

The finding that the CX group did not improve their PE skills over the course of the clerkship, despite performing at least forty physical examinations ${ }^{(11)}$ and $60 \%$ reporting that they received instruction on physical examination, indicates the need to develop teaching sessions that incorporate an assessment of skills acquisition/competence of the participating students. It is 
also of concern that our $3^{\text {rd }}$ year students had similar entry-level skills in abdominal examination irrespective of whether they were at the beginning or end of the 3rd year. The PE maneuvers illustrated by The Infant Patient Encounter program are not unique to pediatrics, or indeed to infants therefore we can assume that the students who came to the pediatric clerkship late in the year had not learned theses basic skills on the other clerkships. Errors were made on all checklist items and these errors of technique would have precluded eliciting physical findings if findings were present. Many students also omitted parts of the examination altogether. The commonest errors were incorrect palpation technique, not using facilitating maneuvers, not exposing the part to be examined and standing on the left side instead of the right side of the patient. These observations highlight the need for consistent, structured teaching with rigorous evaluation coordinated across the core clerkships. It would also be interesting to know if students "carry over" skills that were learned from the pediatric program into the surgery, medicine, ob/gyn and family medicine clerkships and if the program might be used to teach PE in these traditionally "adult" clerkships.

Since completing this study we have experimented with using the physical examination program in different ways. We are currently incorporating it into the PE section of a clinical skills day ${ }^{(12)}$ and are considering selecting specific video and animation clips from the PE for students to load onto required handheld devices (PalmOS PDA), so that they may refer to them during the clerkship. This would compliment the Patient Encounter Log System, used by students during all of the core clerkships at Jefferson Medical College to track the patients they have seen, the skills they have performed, and the procedures they have done. 


\section{References:}

1. Lane JL, Gottlieb RP. Structured clinical observations: a method to teach clinical skills with limited time and financial resources. Pediatrics 2000;105(4 Pt 2):973-977.

2. Lane JL, Gottlieb RP. Improving the interviewing and self-assessment skills of medical students: is it time to readopt videotaping as an educational tool? Ambulatory Pediatrics 2004;4(3):244-248.

3. Jefferson Publishing. (n.d.). Retrieved November 17, 2004, from http://jeffline.jefferson.edu/press/

4. Adler MD, Johnson KB. Quantifying the literature of computer-aided instruction in medical education. Academic Medicine 2000;75(10):1025-1028.

5. Moberg TF, Whitcomb ME. Educational technology to facilitate medical students' learning: background paper 2 of the medical school objectives project. Academic Medicine 1999;74(10):1146-1150.

6. Barzansky B, Jonas HS and Etzel SI. Educational programs in US medical schools, 19992000. JAMA 2000;284(9):1114-1120.

7. Vogel M, Wood DF. Love it or hate it? Medical students' attitudes to computer-assisted learning. Med Educ 2002;36(3):214-215.

8. Carroll AE, Schwartz MW. A comparison of a lecture and computer program to teach fundamentals of the Draw-a-Person test. Arch Pediatr Adolesc Med 2002;156(2):137-140.

9. Desch LW, Esquivel MT and Anderson SK. Comparison of a computer tutorial with other methods for teaching well-newborn care. American Journal of Diseases of Children 1991;145(11):1255-1258. 
10. Steele DJ, Johnson Palensky JE, Lynch TG, Lacy NL and Duffy SW. Learning preferences, computer attitudes, and student evaluation of computerised instruction. [see comment]. Med Educ 2002;36(3):225-232.

11. Rattner SL, Louis DZ and Rabinowitz C, et al. Documenting and comparing medical students' clinical experiences. JAMA 2001;286(9):1035-1040.

12. Lane J.L. Starting students off on the right foot? The pediatric clinical skills day at Jefferson Medical College. Presented at the Council on Medical Student Education in Pediatrics (COMSEP) annual meeting. 2004

The authors wish to thank the following people for their support during the study, data analysis, and editing. Your continued help is very much appreciated.

Chris Braster

Sybil Fullard

Mohammadreza Hojat

Gail Leone

Elizabeth Mikita

Edward Taywea 